\title{
On the nilpotency index of the radical of a group algebra
}

\author{
By Kaoru Motose and Yasushi Ninomiya
}

Throughout the present note, $K$ will represent an algebraically closed field of characteristic $p>0$. In case $G$ is a $p$-solvable group of order $p^{a} m$ $(a \geqq 1, p \nmid m)$, concerning the nilpotency index $t(G)$ of the radical $J(K G)$ of the group algebra $K G$, D. S. Passman [4; Th. 1.6], Y. Tsushima [5; Th .2] and D. A.R. Wallace [7; Th. 3.3] have obtained the following:

$$
p^{a} \geqq t(G) \geqq a(p-1)+1 .
$$

In $\S \S 1$ and 2 of the present note, we shall investigate when $t(G)=p^{a}$ or $t(G)=a(p-1)+1$, where $G$ is a $p$-solvable group of order $p^{a} m(a \geqq 1$, $p \nmid m)$. Furthermore, as an application of Th. 1, we shall present a characterization of a finite group $G$ with $t(G)=[J(K G): K]+1$ (Th. 2).

1. We shall begin our study with the following:

THEOREM 1. If $G$ is a p-group of order $p^{a}$, then there holds the following :

(1) $t(G)=a(p-1)+1$ if and only if $G$ is elementary abelian.

(2) $t(G)=p^{a}$ if and only if $G$ is cyclic.

Proof. (1) Following [3], we consider the $\Re$-series of $G$ :

$$
G=\Re_{1} \supseteq \Re_{2} \supseteq \cdots \supseteq \Re_{t(\theta)}=1,
$$

where $\Re_{2}=\left\{x \in G \mid 1-x \in J(K G)^{\lambda}\right\}$. Then, every $\Re_{2}$ is a characteristic subgroup of $G$ and $\Re_{2} / \Re_{\lambda+1}$ is an elementary abelian group of order $p^{a_{\lambda}}$. By [3; Th. 3.7], we have $t(G)=\sum_{2} \lambda d_{2}(p-1)+1$. If $t(G)=a(p-1)+1$ then $\sum_{\lambda} \lambda d_{\lambda}=a$. Combining this with $\sum_{\lambda} d_{\lambda}=a$, we readily obtain $d_{1}=a$ and $d_{\lambda}=0(\lambda \neq 1)$, namely, $G$ is elementary abelian. The converse is obvious by [3; Th. 6.2].

(2) Suppose $t(G)=p^{a}$. If $\Phi(G)$ is the Frattini subgroup of $G$, then [7; Th. 2.4] yields $|G|=t(G) \leqq t(\Phi(G)) \cdot t(G / \Phi(G)) \leqq|\Phi(G)| \cdot|G / \Phi(G)|=|G|$, whence it follows $t(G / \Phi(G))=|G / \Phi(G)|=p^{b}(b \leqq a)$. Since $G / \Phi(G)$ is elementary abelian, $t(G / \Phi(G))=b(p-1)+1$ by $(1)$. Hence, $p^{b}=|G / \Phi(G)|=$ $t(G / \Phi(G))=b(p-1)+1$, which means $b=1$ and $G / \Phi(G)$ is cyclic. Now, as is well-known, $G$ is cyclic. Concerning the converse, there is nothing to prove.

In what follows, $G_{p}$ will represent a Sylow $p$-subgroup of $G$. 
Corollary 1. Let $G$ be a p-solvable group of order $p^{a} m(a \geqq 1, p \nmid m)$. Then there holds the following:

(1) If $G$ has p-length 1 and $t(G)=a(p-1)+1$ then $G_{p}$ is elementary abelian, and conversely.

(2) If $G$ has p-length 1 and $t(G)=p^{a}$ then $G_{p}$ is cyclic, and conversely.

Proof. Since $G$ is a $p$-solvable group of $p$-length $1, G_{p}$ is normal or $G$ contains a normal $p$-nilpotent subgroup $H$ with $p \nmid(G: H)$. In either case, we have $t(G)=t\left(G_{p}\right)$ by [2; Th. 2]. Our assertions are therefore obvious by Theorem 1 .

The next contains [7; Th. 3.4].

Corollary 2. Let $G$ be a p-solvable group of order $p^{a} m(a \geqq 1, p \nmid m)$. If either $p^{a}=3$ or $p^{a}=4$ and $G_{2}$ is elementary abelian, then $t(G)=3$, and conversely.

2. Throughout the present section, $G$ will represent the symmetric group of degree 4, and $K$ an algebraically closed field of characteristic 2. Obviously, $G$ is a solvable group whose 2-length $>1$ and whose any Sylow 2 -subgroup is not elementary abelian. However, the proposition stated below says that $t(G)=4(=a(p-1)+1)$.

Let $G_{i}$ be the stabilizer of a letter $i$ and $\hat{S}=\sum_{x \in S} x(\in K G)$ for any subset $S$ of $G$.

LEMMA 1. $\widehat{G}_{i} x \widehat{G}_{j}=0$ for every $x \in G$.

PROOF. Since $\widehat{G}_{i} x \widehat{G}_{j} x^{-1}=\widehat{G}_{i} \widehat{G}_{x(j)}$, it suffices to prove that $\widehat{G}_{i} \widehat{G}_{j}=0$. In case $i=j, \hat{G}_{i}^{2}=6 \hat{G}_{i}=0$. While, if $i \neq j$ and $\{1,2,3,4\}=\{i, j\} \cup\{k, l\}$, then $\widehat{G}_{i}=\widehat{G}_{i}(k, l), \widehat{G}_{i}(k, i)=\widehat{G}_{i}(k, l, i)$ and $\widehat{G}_{i}(l, i)=\widehat{G}_{i}(k, i, l)$. Hence $\widehat{G}_{i} \widehat{G}_{j}=\widehat{G}_{i}(1+$ $(k, l)+(k, i)+(k, l, i)+(l, i)+(k, i, l))=2 \widehat{G}_{i}+2 \widehat{G}_{i}(k, i)+2 \widehat{G}_{i}(l, i)=0$.

Proposition. (1) $J(K G)=K \hat{G}_{1} \oplus J(K V) K G$, where $V$ is the Klein's four group contained in $G$.

(2) $t(G)=4$.

Proof. (1) Since $V$ is a normal 2-subgroup of $G, J(K(G / V)) \cong$ $J(K G) / J(K V) K G$. Now, $G / V$ (naturally isomorphic to $G_{1}$ ) is isomorphic to the symmetric group of degree 3 , and then $J(K(G / V))=K \widehat{G / V}$ by [6; Th. 2]. Moreover, noting that $\hat{G}_{1}$ is an element of $J(K G)$ not contained in $J(K V) K G$, we readily obtain (1).

(2) Since $J(K V)^{2}=K \hat{V}$, we have $J(K G)^{2}=\left(K \widehat{G}_{1}+J(K V) K G\right)^{2}=\left(K \widehat{G}_{1}\right)^{2}$ $+J(K V)^{2} K G+\widehat{G}_{1} J(K V)+J(K V) \widehat{G}_{1}=\hat{V} K G+\widehat{G}_{1} J(K V)+J(K V) \hat{G}_{1}$. Noting further that $\hat{G}_{1} J(K V) \hat{G}_{1}=0, \hat{G}_{1}^{2}=0$ (Lemma 1$), \hat{V} J(K V)=0$ and that $\hat{V}$ is a central element of $K G$, we obtain $J(K G)^{4}=\left(\hat{V} K G+\widehat{G}_{1} J(K V)+J(K V) \widehat{G}_{1}\right)^{2}$ 
$=(\hat{V} K G)^{2}+\left(\hat{G}_{1} J(K V)\right)^{2}+\left(J(K V) \hat{G}_{1}\right)^{2}+\hat{V} \hat{G}_{1} J(K V)+\left(\hat{G}_{1} J(K V) \hat{V}\right) K G+$ $\widehat{G}_{1} J(K V)^{2} \widehat{G}_{1}+J(K V) \widehat{G}_{1}^{2} J(K V)+\hat{V} K G\left(J(K V) \hat{G}_{1}\right)+\left(J(K V) \hat{G}_{1} \hat{V}\right) K G=0$. Hence, $t(G) \leqq 4=3(2-1)+1$, whence it follows $t(G)=4$.

3. Let $G$ be an arbitrary finite group such that $p$ is a divisor of $|G|$, and $\left\{e_{i j} \mid 1 \leqq i \leqq s, 1 \leqq j \leqq f(i)\right\}$ a set of orthogonal primitive idempotents of $K G$ with $1=\sum_{i, j} e_{i j}$ such that $K G e_{i j} \cong K G e_{i^{\prime} j^{\prime}}$ if and only if $i=i^{\prime}$. Let $e_{i}=e_{i 1}$ $(1 \leqq i \leqq s), K G e_{1} / J(K G) e_{1}$ a trivial $K G$-module, and $C=\left(c_{k l}\right)$ the Cartan matrix of $G$. In this section, we shall investigate when $t(G)=[J(K G): K]+1$. To our end, a couple of lemmas will be needed. +1 .

LEMmA 2. $t(G) \leqq \max _{k}\left\{\sum_{l} c_{k l}\right\} \leqq \max _{k}\left\{\left[J(K G) e_{k}: K\right]+1\right\} \leqq[J(K G): K]$

Proof. Since $\sum_{l} c_{k l}$ coincides with the length of the composition series of an indecomposable $K G$-module $K G e_{k}, \sum_{l} c_{k l} \leqq\left[J(K G) e_{k}: K\right]+1$. Thus, $t(G) \leqq \max _{k}\left\{\sum_{l} c_{k l}\right\} \leqq \max _{k}\left\{\left[J(K G) e_{k}: K\right]+1\right\} \leqq[J(K G): K]+1$ (cf. [7 ; Lemma 4.2]).

LEMMA 3. The following conditions are equivalent:

(1) $[J(K G): K]=\max _{k} \cdot\left\{\sum_{\imath} c_{k l}\right\}-1$.

(2) $C=\operatorname{diag}\left(p^{a}, 1, \cdots, 1\right)$.

(3) $[J(K G): K]=p^{a}-1$.

(4) $G$ is either a p-group or a Frobenius group with a complement $G_{p}$.

Proof. (2), (3) and (4) are equivalent by the proof of [6; Th. 2]. Hence, it remains only to prove that (1) implies (2). Assume that $[J(K G): K]$ $=\max _{k}\left\{\sum_{\imath} c_{k l}\right\}-1$. Then, by Lemma 2, $1+\sum_{k, j}\left[J(K G) e_{k j}: K\right]=1+[J(K G): K]$ $=\max _{k}\left\{\left[J(K G) e_{k}: K\right]+1\right\}$. Since $\left[J(K G) e_{1}: K\right] \geqq p^{a}-1$ (cf. [1; p. 562]), it follows that $J(K G)=J(K G) e_{1}$ and $J(K G) e_{k}=0$ for $k \neq 1$. Therfore, $C=$ $\operatorname{diag}\left(c_{11}, 1, \cdots, 1\right)$. This means that the first block contains only one irreducible modular character, and hence $c_{11}=\left[J(K G) e_{1}: K\right]=p^{a}$ (cf. [1; p. 587]).

Now, we shall conclude our study with the following:

Theorem 2. $t(G)=[J(K G): K]+1$ if and only if $G$ is either a cyclic p-group or a Frobenius group with a cyclic complement $G_{p}$.

Proof. If $G$ is either a cyclic $p$-group or a Frobenius group with a cyclic complement $G_{p}$, then $t(G)=t\left(G_{p}\right)=\left|G_{p}\right|=[J(K G): K]+1$ (cf. [2; Th. 2] and [6; Th. 2]). Conversely, if $t(G)=[J(K G): K]+1$ then, by Lemmas 2 and $3, G$ is either a $p$-group or a Frobenius group with a complement $G_{p}$. Moreover, we have $t\left(G_{p}\right)=t(G)=[J(K G): K]+1=\left|G_{p}\right|$, so that 
$G_{p}$ is cyclic by Theorem 1 .

Shinshu University

Okayama University

\section{References}

[1] R. BRAuER and C. NESBitT: On the modular characters of groups, Ann. of Math. 42 (1941), 556-590.

[2] R. J. ClARKE: On the radical of the group algebra of a p-nilpotent group, J. Australian Math. Soc. 13 (1972), 119-123.

[3] S. A. JENNINGS: The structure of the group ring of a $p$-group over a modular field, Trans. Amer. Math. Soc. 50 (1941), 175-185.

[4] D. S. PAssman: Radicals of twisted group rings, Proc. London Math. Soc. 20 (1970), 409-437.

[5] Y. Tsushima: Radicals of group algebras, Osaka J. Math. 4 (1967), 179-182.

[6] D. A. R. WAllace: Note on the radical of a group algebra, Proc. Cambridge Phil. Soc. 54 (1958), 128-130.

[7] D. A. R. WALLACE: Lower bounds for the radical of the group algebra of a finite $p$-soluble group, Proc. Edinburgh Math. Soc. 16 (1968/69), 127-134.

(Received June 4, 1974) 